\title{
Immediate antibiotics were beneficial in children with acute otitis media who had fever and vomiting
}

\author{
Little P, Gould C, Moore M, et al. Predictors of poor outcome and benefits from antibiotics in children with acute otitis \\ media: pragmatic randomised trial. BMJ 2002;325:22-25.
}

\section{QUESTION: In children with acute otitis media and who are at risk of a poor outcome, what is the comparative effectiveness of immediate and delayed antibiotics?}

\section{Design}

Randomised \{allocation concealed $*\}$, \{unblinded $\} \nmid *$ trial with 3 days of follow up. This study was a secondary analysis of a previously published randomised controlled trial\$.

\section{Setting}

Primary care setting in the Southampton area, UK.

\section{Patients}

285 children between 6 months and 10 years of age with acute otitis media and information on symptom duration. Exclusion criteria were pink drum alone, otitis media with effusion and chronic suppurative otitis media, serious chronic disease, use of antibiotics in previous 2 weeks, previous complications, or systemic ill health. Follow up was $90 \%$.

\section{Intervention}

Children were assigned to immediate antibiotics (amoxicillin or erythromycin) $\{\mathrm{n}=135\} \dagger$ or delayed antibiotics (wait $72 \mathrm{~h}$ after seeing the doctor before using prescription) $\{\mathrm{n}=150\} \uparrow$. If the child had severe otalgia, fever after 72 hours, or discharge lasted $\geq 10$ days, parents were advised to use antibiotics.

\section{Main outcome measures}

Episodes of distress and night disturbance by day 3 and days of crying were reported by parent.

Source of funding: NHS Regional

Research and

Development.

For correspondence:

Dr P Little, University

of Southampton,

Southampton, UK.

Psl3@soton.ac.uk

\section{Conclusions}

In children with acute otitis media and fever or vomiting, immediate antibiotic treatment was more beneficial than delayed antibiotic treatment for reducing distress, night disturbance, and crying. Among those without fever or vomiting, immediate and delayed antibiotic treatment did not differ.

*See glossary.

Information provided by author.

\$Little P, Gould C, Williamson I, et al. BMJ 2001;322:336-42.

\section{COMMENTARY}

A vexing problem with otitis media research is the lack of agreement over the diagnostic definition of acute otitis media. ${ }^{1}$ No widely accepted gold standard for the diagnosis of this condition exists, which means there is no way to determine which combination of clinical findings provides the most accurate diagnosis.

The most practical definition of acute otitis media may be the combination of clinical findings that best identifies children who will benefit most from antibiotics. Little $e t$ al have taken an important first step towards finding such a definition. They identified several symptoms and signs at presentation that were associated with more severe morbidity during follow up. They then assessed the benefit of immediate antibiotic treatment in subgroups of children with these predictive clinical findings.

In their initial study, the authors found a modest benefit of immediate antibiotic treatment similar to those found in other studies. ${ }^{2}$ However, in this secondary analysis, they show that children with acute otitis media accompanied by fever or vomiting had a more substantial improvement from antibiotics, whereas children with neither vomiting nor fever did not benefit from antibiotics.

Because this is a secondary analysis and multiple symptoms and signs were evaluated, it is possible that the findings were because of chance, despite the statistical significance. On the other hand, the authors used very low antibiotic doses, and perhaps using high dose amoxicillin would result in a greater treatment benefit. Either way, other studies are needed to confirm the present findings. Such studies should also evaluate individual otoscopic findings to establish diagnostic criteria that best identify children who will benefit from antibiotic treatment. Such research could lead to substantial improvement in our ability to decrease unnecessary antibiotic use while maintaining maximum benefit to children with acute otitis media.

Michael B Aldous, MD, MPH University of Arizona Tucson, Arizona, USA

1 Hayden GF. Acute suppurative otitis media in children. Diversity of clinical diagnostic criteria. Clin Pediatr (Phila) 1981;20:99-104.

2 Little P, Gould C, Williamson I, et al. Pragmatic randomised controlled trial of two prescribing strategies for childhood acute otitis media. BMJ 2001;322:336-42. 